\title{
A Novel Algorithm for Fault Type Fast Diagnosis in Overhead Transmission Lines Using Hidden Markov Models
}

\author{
M. Jannati*, S. Jazebi*, B. Vahidi ${ }^{\dagger}$ and S. H. Hosseinian*
}

\begin{abstract}
Power transmission lines are one of the most important components of electric power system. Failures in the operation of power transmission lines can result in serious power system problems. Hence, fault diagnosis (transient or permanent) in power transmission lines is very important to ensure the reliable operation of the power system. A hidden Markov model (HMM), a powerful pattern recognizer, classifies events in a probabilistic manner based on fault signal waveform and characteristics. This paper presents application of HMM to classify faults in overhead power transmission lines. The algorithm uses voltage samples of one-fourth cycle from the inception of the fault. The simulation performed in EMTPWorks and MATLAB environments validates the fast response of the classifier, which provides fast and accurate protection scheme for power transmission lines.
\end{abstract}

Keywords: EMTPWorks, Transient and permanent faults, Secondary arc, Hidden Markov model (HMM)

\section{Introduction}

Transmission networks, being the most essential part of the power system, establish connection between production and consumption units. Nowadays, providing consumer requirements and preventing long interruptions are of great importance due to increasing developments in electrical power applications. Because of the widespread use and geographical conditions of transmission line regions, power systems are exposed to various faults. These faults can cause the disabling of transmission lines and interruption in power provision in some parts of the power network.

Nearly $80 \%$ of faults in an EHV transmission lines are transient [1]. The most prevalent potential of transient faults is lightning and transmission line switching, among others. This transient faults can be cleared by temporarily opening the faulty phase. In this type of faults, transmission line can be energized by a single-pole autoreclosure. This strategy can improve the reliability and stability of power system [2]. Other types of faults, including permanent faults such as a wire's scrapping, are not clearable by auto-reclosure. The traditional autoreclosure with a fixed setting for dead time is not recommended in transmission lines. Therefore, a novel algorithm for fault type fast diagnosis in high-voltage transmission lines using a hidden Markov model (HMM) is presented in this paper.

Various alternatives have been performed for fault type

$\dagger$ Corresponding Author: Department of Electrical Engineering, Amirkabir University of Technology, Tehran, Iran (vahidi@aut.ac.ir)

* Department of Electrical Engineering, Amirkabir University of Technology, Tehran, Iran (mohsen.jannati@aut.ac.ir, saeed_3j@aut.ac.ir, hosseinian@aut.ac.ir)

Received: September 18, 2010; Accepted: September 19, 2011 diagnosis [3-15]. For instance, the RMS of faulty phase voltage was used in [5]. The current high-frequency components of one of the two robust phases that contain the load current were used in [6]. The algorithm of [7] was based on the low-frequency components of the faulted phase voltage. The method of [8] used the fundamental component of zero sequence power in both line terminals. Neural network was presented in [9] to carry out the identification task. Some references, such as [10], have also used wavelet transform, and some, such as [11], combined it with neural network. A method based on fuzzy logic was proposed in [12]. In [13], THD of the faulted phase voltage was used to distinguish transient and permanent faults in transmission lines. In [14], the voltage waveform at the sending end of the power transmission line was analyzed during the fault, and an adaptive linear neuron was then trained online to identify transient and permanent faults.

HMM, a powerful pattern recognizer, classifies the events in a probabilistic manner based on fault signal waveform and characteristics [15]. The HMM ability to solve small sample, nonlinear, and high-dimensional pattern problems make this algorithm a powerful choice for application in power system disturbance classifications [ $[16$, 17], partial discharge de-noising [18], accidents identification and decision making in power plants [19], modeling and forecasting electrical power markets [20-22], and power transformer fault diagnosis based on dissolved gas analysis [23]. In [24, 25], HMMs were specially applied to power transformer differential protection. HMM requires only a small amount of computation for decision making (classification) after training; hence, it can be implemented for online applications. This advantage makes the HMM very attractive for fault diagnosis. The 
probabilistic inherent of HMM makes it flexible for different operating conditions without considering any deterministic index. This paper presents a novel application of HMMs for fast and accurate reclosing command.

A typical transmission line is simulated in EMTPWorks and executed in extensive conditions. The post fault voltage signal for the faulted phase is retrieved at the sending or receiving end at a sampling frequency of 10 $\mathrm{kHz}$. The moving window width was taken at one-fourth power frequency cycle. The simulated signals from EMTPWorks are then fetched to the classifier, which is coded in MATLAB environment. Simulation results show that HMM can distinguish between transient and permanent faults for all possible states.

\section{Basic Theory of Discrete HMM}

HMM is a classification algorithm with probabilistic phenomena. This algorithm is adjustable to novel data where there is no complete information about the source from which the signals are generated. The difference between Markov chain and an HMM lies in observations.

In HMM, observations are probabilistic function of the related state and its probability distribution function. This feature is an advantage of HMM, which provides more flexibility to overcome uncertainties in a real-world power system. The structure of HMM is discussed in the following.

An HMM can be defined as $\lambda=(N, M, \pi, A, B)$, where $N$ is the number of states, $M$ is the number of distinct observation symbols per state, $\pi$ is the initial state distribution vector, and $A$ and $B$ are the state transition probability and observation probability matrices respectively. The elements of matrix $A, a_{i j}$, is the transition probability from state $i$ to state $j$, which are defined in Eqs. (1) and (2). In these equations, $q_{t}$ is the actual state $S$ at time $t$. An example of a three-state HMM with three observation symbols in each state is illustrated in Fig. 1.

$$
\begin{gathered}
a_{i j}=P\left[q_{t+1}=S_{j} \mid q_{t}=S_{i}\right], 1 \leq i, j \leq N \\
a_{i j} \geq 0, \quad \sum_{j=1}^{N} a_{i j}=1
\end{gathered}
$$

The elements of matrix $B, b_{j}(k)$, are defined by Eq. 3, where $V_{k}$ is the $k^{\text {th }}$ observation in the state. Matrix $B$ and vector $\pi$ elements follow the rules presented in Eq. 4 . The HMM training process is identical to find appropriate parameters of $A, B$, and $\pi$.

$$
b_{j}(k)=P\left[O_{t}=V_{k} \mid q_{t}=S_{j}\right], 1 \leq j \leq N, 1 \leq k \leq M
$$

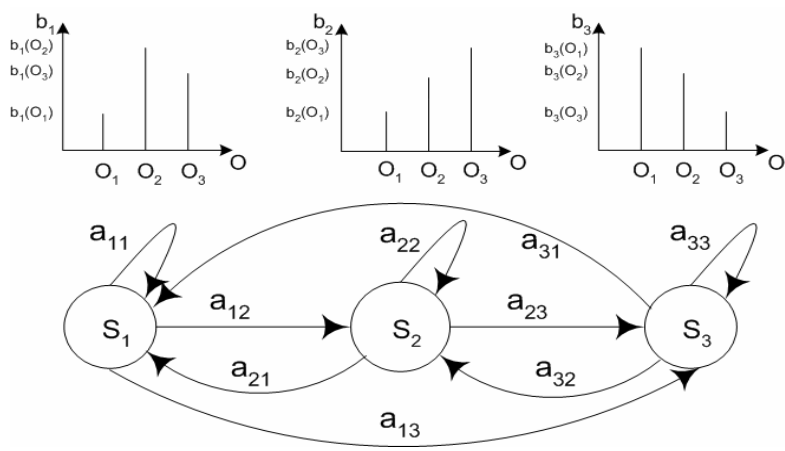

Fig. 1. Schematic of a typical HMM

$$
b_{j}(k) \geq 0, \sum_{k=1}^{M} b_{j}(k)=1, \sum_{i=1}^{N} \pi_{i}=1
$$

where $O_{t}$ indicates observation at time $t$. Eq. (3) calculates the probability of observation $V_{k}$ at time $t$, where $q_{t}=S_{j}$.

\section{Simulated Power Transmission System}

Fig. 2 demonstrates the single-line diagram of a $400 \mathrm{kV}$ and $300 \mathrm{~km}$ power transmission line simulated in the EMTPWorks program.

The input data set of HMM (for transient and permanent faults) is derived from excessive simulations on the above power system. The power transmission line parameters are given as follows.

Positive sequence resistance, inductance, and capacitance are $\mathrm{R}_{1}=0.01526 \Omega / \mathrm{km}, \mathrm{L}_{1}=0.8838 \mathrm{mH} / \mathrm{km}$, and $\mathrm{C}_{1}=0.0126 \mu \mathrm{F} / \mathrm{km}$, respectively; meanwhile, zero sequence resistance, inductance, and capacitance are $\mathrm{R}_{0}=$ $0.04624 \Omega / \mathrm{km}, \mathrm{L}_{0}=2.6563 \mathrm{mH} / \mathrm{km}$, and $\mathrm{C}_{0}=0.0043$ $\mu \mathrm{F} / \mathrm{km}$, respectively. Thevenin's equivalent impedances at the sending and receiving ends are described using mutual coupled R-L circuit: the positive sequence is $R_{1}=0.06 \Omega$ and $\mathrm{L}_{1}=40.03 \mathrm{mH}$, while the zero sequence is $\mathrm{R}_{0}=0.127$ $\Omega$ and $\mathrm{L}_{0}=23.56 \mathrm{mH}$. The distributed line parameter model of EMTPWorks is intentionally selected to simulate the unsymmetrical faults.

The two major types of arcing faults occurring on power transmission lines are permanent and transient faults. A linear resistance is usually an adequate representation for the permanent fault, whereas a nonlinear arc model depicts the transient fault. Various studies in [14] have shown that the dynamic characteristics of the electric arc could be simulated by the following empirical equations:

$$
\begin{aligned}
& \frac{d g}{d t}=\frac{1}{\tau}(G-g) \\
& G=\frac{|i|}{\left(U_{o}+R|i|\right) \ell}
\end{aligned}
$$



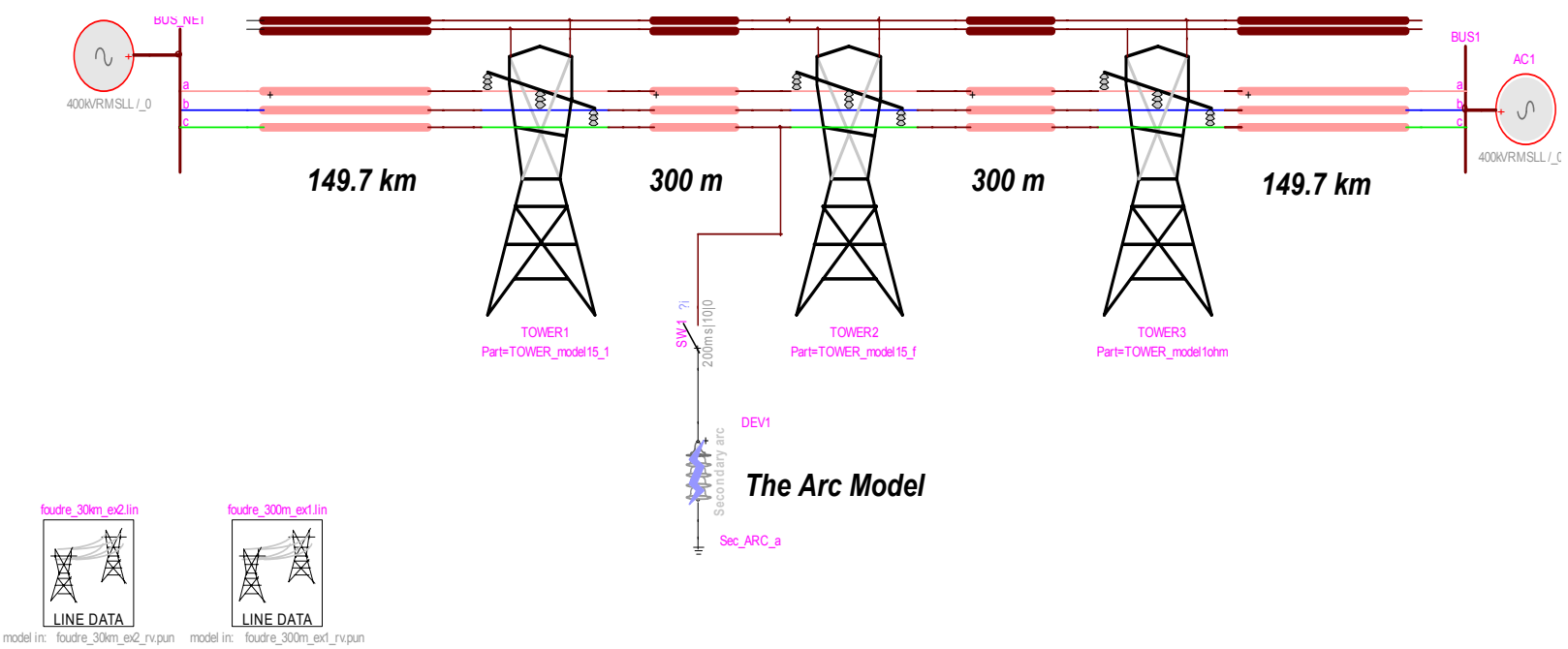

The Arc Model

Fig. 2. The power transmission system under study

where $g_{k}$ is the dynamic arc conductance, $\tau$ is the arc channel time constant, $G$ is the stationary arc conductance, $i$ is the arc current, $U_{o}$ is the arc voltage drop per unit length along the main arc columns, $\ell$ is the arc length, and $R$ is the total resistance of the cathode and anode regions of the arc, which is independent of the current.

The values of $\tau, U_{o}$, and $R$ vary according to the characteristics of the transmission line and other environmental conditions. They could be estimated from high-voltage lab tests. Moreover, the length variation of the arc is approximated by the following equation for low wind velocities of $0-1 \mathrm{~m} / \mathrm{s}$ [14]:

$$
\ell_{s}\left(t-t_{s}\right)= \begin{cases}10 \times \ell_{s o} \times\left(t-t_{s}\right) & t>0.1+t_{s} \\ \ell_{s o} & t \leq 0.1+t_{s}\end{cases}
$$

where $\ell_{s o}$ is the primary arc length and $t_{s}$ is the time in which circuit breakers are opened.

Using Eqs. (5)-(7), a nonlinear time-dependent dynamic resistance representing the primary and secondary arcs is simulated in this paper. The modeled arc is used for autoreclosure studies.

The sampling process for the voltage waveform of the faulted phase commences when an SLG fault (transient or permanent) occurs and the power breakers of the faulted phase open. Consequently, the harmonics of the voltage waveform of the faulted phase should be derived using the calculation of Fast Fourier Transform (FFT) or An ADAptive Linear Neuron (ADALINE) neural network.

Fig. 3 demonstrates the voltage at the sending end of the high-voltage transmission line when a transient fault occurs at $\mathrm{t}=0.160$ seconds. In this case, an SLG fault occurred on phase $\mathrm{C}$, and secondary arc started at $\mathrm{t}=0.210$ seconds and voiding phase $\mathrm{C}$. The secondary arc is extinguished after 0.240 seconds from the start.

The voltage waveform of the faulted phase in the

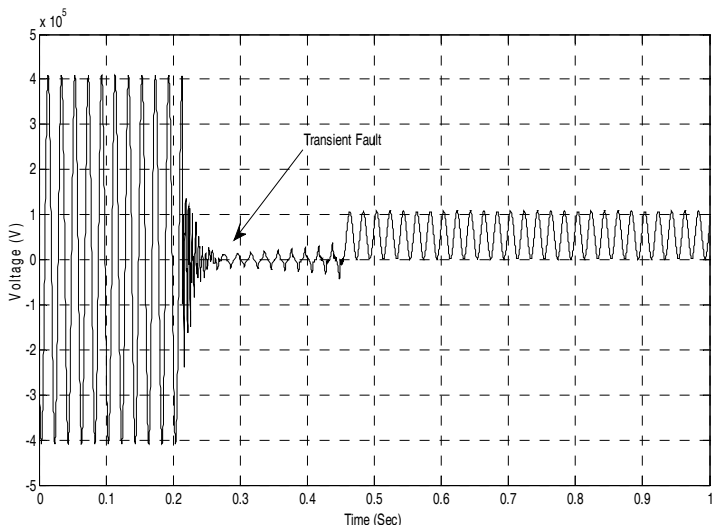

Fig. 3. Voltage waveform corresponding to the inverse of the fundamental harmonic

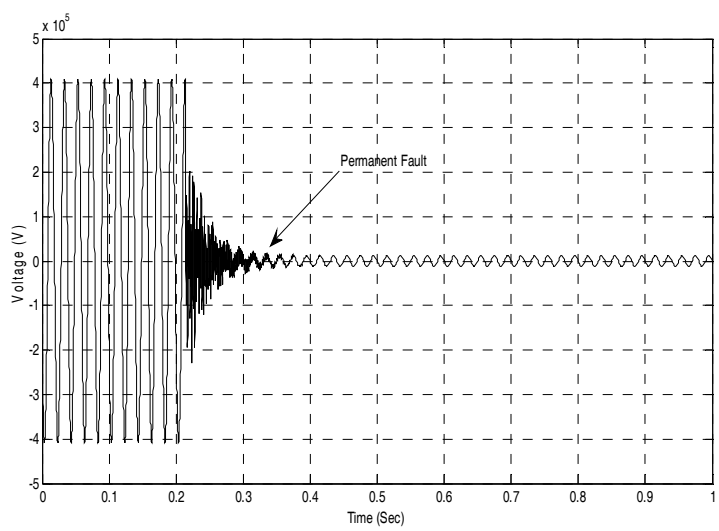

Fig. 4. Voltage waveform corresponding to the inverse of the fundamental harmonic

secondary arc period is distorted compared to the sinusoidal waveform in the pre-fault condition. In addition, the voltage of the faulted phase starts to increase by satisfying the secondary arc. Fig. 4 shows the voltage waveforms during the permanent fault. 


\section{Simulation and Classification of Fault Type}

\subsection{HMM training process}

HMM classifies faults by comparing the maximum likelihood probability of the fault signal for trained models. Thus, an HMM model should be trained for each kind of fault. In the HMM training process, the parameters are recalculated iteratively to statistically match to a group of training signals. The following section describes the training data set selection and training scheme.

It is necessary to build two separate HMM blocks for transient and permanent faults. The HMM block inputs are observation vectors $O=O_{1}, O_{2}, \ldots . O_{T}$, which are feature signal samples (of faulted waveform). In the training process, the maximum likelihood probability denoted by Eq. (8a) should be maximized indirectly using the logarithm of the above probability (Loglik), which is presented in Eq. (8b).

$$
\begin{gathered}
P(O \mid \lambda)=\sum_{\text {All } Q} P(O \mid Q, \lambda) P(Q, \lambda) \\
\operatorname{Loglik}=\log (P(O \mid \lambda))
\end{gathered}
$$

where $Q=q_{1}, q_{2}, \ldots q_{T}$ is a fixed state sequence and $T$ is the number of observations. The Baum-Welch algorithm is used in the problem $[15,17]$. Baum-Welch first defines $\gamma_{t}(i, j)$ (the posteriori probability of transitions being in state $i$ at time $t$ and making a transition to state $j$ at $t+1$, given the observation sequence). It can be computed as Eq. 9 , and the variable $\gamma_{t}$ (the posteriori probability of being in state $i$ at time $t$, given the observation sequence) is defined by Eq. 10 .

$$
\begin{aligned}
& \gamma_{t}(i, j)=P\left(S_{t}=i, S_{t+1}=j \mid O, \lambda\right)= \\
& \frac{\alpha_{t}(i) a_{i j} b_{j}\left(O_{t+1}\right) \beta_{t+1}(j)}{P(O \mid \lambda)}= \\
& \frac{\alpha_{t}(i) a_{i j} b_{j}\left(O_{t+1}\right) \beta_{t+1}(j)}{\sum_{k \in Q_{F}} \alpha_{T}(k)} \\
& \gamma_{t}(i)=P\left(s_{t}=i \mid O, \gamma\right)=\frac{\alpha_{t}(i) \beta_{t}(i)}{P(O \mid \lambda)} \\
& =\frac{\alpha_{t}(i) \beta_{t}(i)}{\sum_{k \in Q_{F}} \alpha_{T}(k)}
\end{aligned}
$$

where $\alpha_{t}(i)$ is the forward variable for model $\lambda$. This is the probability of the partial observation sequence $O$ (until time $t$ ) and state $S_{i}$ at time $t$. Another parameter is $\beta_{t}(i)$, which is a backward variable that refers to the probability of the partial observation sequence from $t+1$ to the end, given $S_{i}$ at time $t$ and the model $\lambda . Q_{F}$ is a set of final states.

$$
\begin{gathered}
\alpha_{t}(i)=P\left(O_{1}, O_{2}, \cdots, O_{t}, q_{t}=S_{i} \mid \lambda\right) \\
\beta_{t}(i)=P\left(O_{t+1}, O_{t+2}, \cdots, O_{T} \mid q_{t}=S_{i}, \lambda\right)
\end{gathered}
$$

If $\alpha_{1}(i)=\pi_{i} b_{i}\left(O_{1}\right)$, then $\alpha$ can be calculated as follows:

$$
\alpha_{t+1}(j)=\left[\sum_{i=1}^{N} \alpha_{t}(i) a_{i j}\right] b_{j}\left(O_{t+1}\right)
$$

If $\beta_{T}(i)=1$ (initialization), then the following holds true:

$$
\beta_{t}(i)=\sum_{j=1}^{N} a_{i j} b_{j}\left(O_{t+1}\right) \beta_{t+1}(j)
$$

Parameters $\bar{a}_{i j}, \bar{b}_{j}, \bar{\pi}_{i}$ of the re-estimated new model $\bar{\lambda}$ can be computed as follows:

$$
\begin{array}{r}
\bar{a}_{i j}=\frac{\sum_{t=1}^{T-1} \gamma_{t}(i, j)}{\sum_{t=1}^{T-1} \gamma_{t}(i)} \\
\bar{b}_{j}(k)=\frac{\sum_{t \in O_{t}=v_{k}} \gamma_{t}(j)}{\sum_{t=1}^{T} \gamma_{t}(j)} \\
\bar{\pi}_{i}=\gamma_{1}(i)
\end{array}
$$

This re-estimation continues by replacing $\bar{\lambda}$, instead of $\lambda$, until $P(O / \lambda)$ converges to a maximum.

\subsection{Fault identification}

The proposed algorithm flowchart is depicted in Fig. 5. When an SLG fault occurs, a windowed fundamental harmonic of the faulted voltage waveform of about a quarter of a cycle ( $5 \mathrm{~ms}$ for $50 \mathrm{~Hz}$ frequency) is sampled. While signals have been sampled at $10 \mathrm{kHz}$, each window consists of 50 samples.

The identification pre-process starts through the quantization of the input observation signal (faulted signal). In the next stage, the quantized signal is fed into HMM models. The logarithm of the models' output probabilities are then computed in the recognition stage. Recognition or classification means finding the best path in each trained model and selecting the one that maximizes the path probability for a given input observation $O$ and the model $\lambda_{i}=\left(A_{i}, B_{i}, \pi_{i}\right), i=1,2, \ldots D$, where $D$ represents the number of fault types. Therefore, fault model $\lambda^{*}$ should satisfy the following equation: 


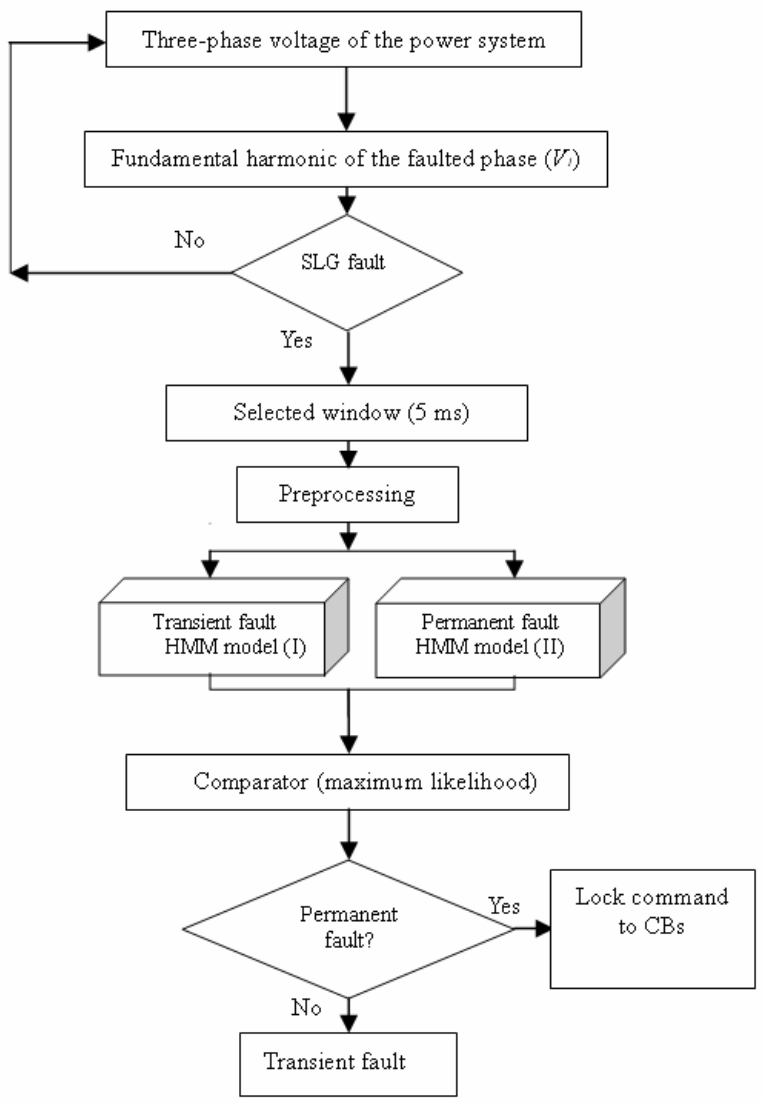

Fig. 5. The proposed algorithm logic

$$
\lambda^{*}=\max _{i}\left[\max _{Q} P\left(Q, O \mid \lambda_{i}\right)\right]
$$

In this stage, the Viterbi algorithm finds the best state sequence $Q[15]$ :

Initialization for all state $\mathrm{i}$ :

$$
\delta_{1}(i)=\pi_{i} b_{i}\left(O_{1}\right), \quad \psi_{1}(i)=0
$$

Recursion from time $\mathrm{t}=2$ to $\mathrm{T}$ and all states $\mathrm{j}$ :

$$
\begin{gathered}
\delta_{t}(j)=\max _{i}\left[\delta_{t-1}(i) a_{i j}\right] b_{j}\left(O_{t}\right) \\
\psi_{t}(j)=\arg _{i} \max \left[\delta_{t-1}(i) a_{i j}\right]
\end{gathered}
$$

Termination:

$$
P^{*}=\max \left[\delta_{T}(i)\right], \quad q_{T}^{*}=\arg \max \left[\delta_{T}(i)\right]
$$

State sequence backtracking from T-1 to 1:

$$
q_{t}^{*}=\psi_{t+1}\left(q_{t+1}^{*}\right)
$$

The maximum likelihood probability $P(O / \lambda)$ could be achieved, given the best path $Q$ and observation $O$ (observation vector of the fault signal). The fault can then be identified by comparing the logarithm of the likelihood probabilities (Loglik) of the models. The model with higher Loglik shows the fault type. The reclosing command, where the transient fault HMM blocks the output, becomes higher; otherwise, the signal will be identified as a permanent fault, resulting in the tripping of the relay.

Two experiments were designed to study the diagnosis ability of HMM. In the first experiment, the effect of the extracted feature in the diagnosis ability of HMM was investigated. In addition, various simulations were performed to ensure the authentic operation of HMM in the classification of faults in the second experiment.

\section{Experiment 1}

HMM is a classification method which is sensitive to selected features like other classification algorithms. Hence, the first experiment in this paper was designed to select the best input data that lead to the best solution.

In this experiment, the number of input data of HMM was 400 (half of them are transient and half are permanent). This data set was generated for three different situations according to the descriptions presented in Table 1. This data set includes voltage waveform of the faulted phase, THD of the voltage waveform, and fundamental harmonic of the voltage waveform $\left(V_{1}\right)$ of the faulted phase. The results of the simulations are given in Fig. 6. It is clear

Table 1. Simulation results for the diagnosis ability of HMM (experiment 1)

\begin{tabular}{c|c|c|c|c}
\hline & Data type & $\begin{array}{c}\text { Number of } \\
\text { transient } \\
\text { faults }\end{array}$ & $\begin{array}{c}\text { Number of } \\
\text { permanent } \\
\text { faults }\end{array}$ & $\begin{array}{c}\text { Diagnosis } \\
\text { ability of } \\
\text { HMM }\end{array}$ \\
\hline Case 1 & $\begin{array}{c}\text { Voltage waveform of } \\
\text { faulted phase }\end{array}$ & 200 & 200 & $\mathbf{7 1 . 5 6 \%}$ \\
\hline Case 2 & $\begin{array}{c}\text { THD of the voltage } \\
\text { waveform of } \\
\text { faulted phase }\end{array}$ & 200 & 200 & $\mathbf{8 3 . 3 8 \%}$ \\
\hline $\begin{array}{c}\text { Fundamental harmonic } \\
\text { of the voltage } \\
\text { waveform of faulted } \\
\text { phase }\end{array}$ & 200 & 200 & $\mathbf{9 9 . 2 \%}$ \\
\hline
\end{tabular}

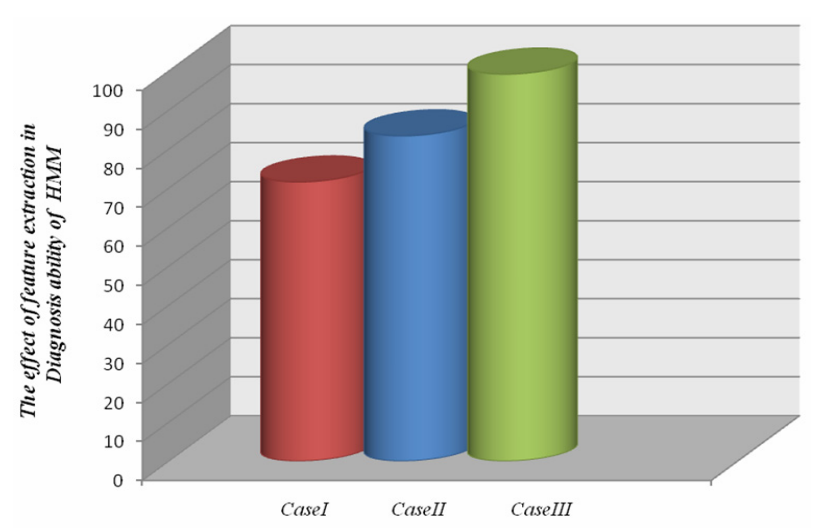

Fig. 6. The illustration of HMM accuracy in experiment 1 
from Fig. 6 that the best input datum (feature) is $V_{1}$.

As a result, $V_{1}$ was chosen as the input datum for the HMM classifier in experiment 2.

\section{Experiment 2}

This experiment was designed to study the diagnosis ability of HMM. Different conditions were considered, as follows:

Case 1:

The input data of HMM consist of 400 sample signals, where 200 are transient faults and the remaining are permanent faults. The data set obtained is composed of SLG faults for different locations in the transmission line. The input data set includes the signal for the faulted phase in sending the end of the transmission line. The classification rate was calculated as $99.2 \%$, which indicates the successful performance of the HMM classifier.

Case 2:

The simulations were performed for different phases and loading conditions. As in the previous case, the number of input data is 400 . A classification accuracy of $97.35 \%$ was achieved in this case.

\section{Case 3:}

To ensure the authentic operation of HMM, a variety of data was chosen for training in this case. In addition to the different fault locations and loading conditions, the transmission line has been equipped by high-speed grounding switches for faster quenching of the secondary arc. The transposed and un-transposed transmission lines were also considered. Moreover, the fault was simulated for both 400 and $765 \mathrm{kV}$ transmission lines. The number of the input data set is the same as in the previous cases. A recognition rate of $95.42 \%$ was achieved in this case. This indicates the accuracy and authenticity of HMM in the classification of fault type in power transmission lines.

In this experiment, three cases were considered, applying various situations in each case. The simulation results presented in Table 2 and Fig. 7 demonstrate the diagnosis ability of HMM for these three cases. Although cases 1 and 2 have more regular considerations than case 3 , the classification rate of the latter is high and is acceptable for such severe conditions.

Table 2. Simulation results for the diagnosis ability of HMM (experiment 2)

\begin{tabular}{c|c|c|c|c|c}
\hline $\begin{array}{c}\text { Number of } \\
\text { transient } \\
\text { faults }\end{array}$ & $\begin{array}{c}\text { Number of } \\
\text { permanent } \\
\text { faults }\end{array}$ & $\begin{array}{c}\text { Different } \\
\text { fault } \\
\text { locations }\end{array}$ & $\begin{array}{c}\text { Use of } \\
\text { auxiliary } \\
\text { device for } \\
\text { fast } \\
\text { quenching } \\
\text { secondary } \\
\text { arc }\end{array}$ & $\begin{array}{c}\text { Different } \\
\text { power } \\
\text { systems } \\
\text { under } \\
\text { study }\end{array}$ & $\begin{array}{c}\text { Diagnosis } \\
\text { ability of } \\
\text { HMM }\end{array}$ \\
\hline 200 & 200 & & - & - & $\mathbf{9 9 . 2 \%}$ \\
\hline 200 & 200 & & & - & $\mathbf{9 7 . 3 5 \%}$ \\
\hline 200 & 200 & & & & $\mathbf{9 5 . 4 2 \%}$ \\
\hline
\end{tabular}

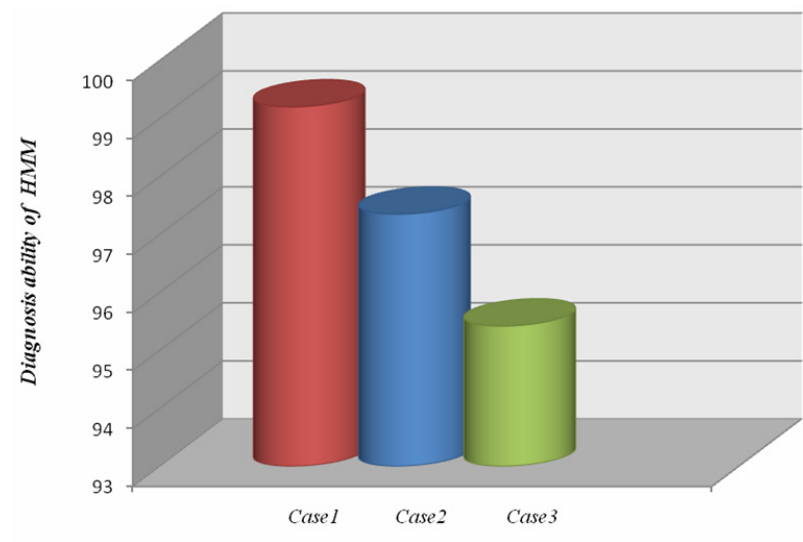

Fig. 7. The illustration of HMM accuracy in experiment 2

\section{Very Fast Protection Scheme for Transmission Line}

Traditional methods such as $[4,13]$ cannot be considered as benchmarks for very fast diagnosis of fault type because they require at least one cycle of power frequency to distinguish between the two types of faults (due to voltage waveform harmonics extraction). In contrast, HMM is a powerful method for statistical classification of data in time domain, which is also independent to harmonics. The simulation results presented in the previous section demonstrate that only one-fourth cycles of power frequency is enough to determine the type of faults in power transmission lines. The proposed HMM-based method is very fast, thereby providing an accurate and reliable protection scheme for protection relaying.

\section{Conclusion}

In this paper, a novel intelligent algorithm was proposed to distinguish between transient and permanent faults in power transmission lines based on HMMs. The HMM ability to solve small sample, nonlinear, and highdimensional pattern problems make this algorithm a powerful choice for application in power system protection. The HMM-based method uses only a data window of onefourth cycle after the fault, thus providing a fast scheme for online diagnosis in power transmission lines. Simulation results in EMTPWorks and MATLAB environments show that the proposed algorithm can accurately identify fault type in high-voltage transmission lines. This reliable and accurate identification scheme can lead to significant advances in power transmission line protection.

\section{References}

[1] C. E. J. Bowler, P. G. Brown, and D.N. walker, "Evaluation of The Effect of Power Circuit Break 
Reclosing Practices on Turbine-Generator Shaft," IEEE Trans. Power App. Syst., vol. PAS-99, pp.17641779, Sept. 1980.

[2] Mohsen Jannati, Behroz Vahidi, Seyed Hossein Hosseinian and Hamid Reza Baghaee, "A Novel Approach for Optimizing Dead Time of Extra High Voltage Transmission Lines" Optimization of Electrical and Electronic Equipment, OPTIM. 11th International Conference on (IEEE), pp.215 - 220, May. 2008.

[3] Z. M. Rajovedic and J. R. Shin, "New One Terminal Digital Algorithm for Adaptive Reclosing and Fault Distance Calculation on Transmission Line," IEEE Trans. Power Del., vol.21, no.3, pp.37-41, July. 2006.

[4] Mohsen Jannati, Behroz Vahidi, Seyed Hossein Hosseinian and Hamid Reza Baghaee, "A New Adaptive Single Phase Auto-Reclosure Scheme for EHV Transmission Lines" Power System Conference, MEPCON. 12th International MiddleEast (IEEE), pp.203 - 207, March. 2008.

[5] Sang-Pil Ahn, Chul-Hwan Kim, R.K. Aggarwal and A.T. Johns, "An Alternative Approach to Adaptive Single Pole Auto-Reclosing in High Voltage Transmission Systems Based on Variable Dead Time Control," IEEE Trans. Power Del., vol.16, pp.676686, Oct. 2001.

[6] Z.Q. Bo, R. K. Aggarwal and A. T. Johns, "A Novel Technique to Distinguish Between Transient and Permanent Fault Based on Detection of Current Transients," Proceeding of 4th International Conference on Advances in Power System Control and Management, pp.217-220, APSCOM-97, Hong Kong, Nov. 1997.

[7] Ge Yaozhang, Sui Fanghai and Xiao Yuan, "Prediction Method for Preventing Single-Phase Reclosing on Permanent Faults," IEEE Trans. Power Del., vol.4, pp.114-121, Jan. 1989.

[8] N. I. Elkalashy, H. A. Darwish, A. M. I. Taalab, M.A. Izzularab, "An Adaptive Single Pole Autoreclosure Based on Zero Sequence Power," Electric Power System Research., vol.77, pp.438-446, 2007.

[9] R. K. Aggarwal, A. T. Johns, Dunn. R.W, Fitton. D.S, "Neural Network based Adaptive Sigle-Pole Autoreclosure Technique for EHV extinguishing time of secondary arc and fault location in PTL Transmission System," IEE Proc- Gener. Transm. Distrib., vol.141, pp.155-160, March. 1994.

[10] Majid Sanaye-Pasand and Ali kadivar, "Design of An Online Adaptive Auto-Reclose Algorithm for $\mathrm{HV}$ Power transmission lines," IEEE Power India Conference, Apr. 2006.

[11] I. K. Yu, Y. H. Song, "Wavelet Transform and Neural Network Approach to Developing Adaptive SinglePole Auto-Reclosing Schemes for EHV Transmission Systems," IEEE Power Engineering Review, pp.6264, Nov. 1998.
[12] Xiangning Lin, Pei Liu, "Method of Distinguishing Between Instant and Permanent Fault of Transmission Lines Based on Fuzzy Decision," IEEE Catalog No. 98EX137, vol.2, pp.455-460, 1998.

[13] Zoran M. Radojevic and Joong-Rin Shin, "New Digital Algorithm for Adaptive Reclosing Based on The Calculation of The Faulted Phase Voltage Total Harmonic Distortion Factor," IEEE Trans. Power Del., vol.22, pp.37-41, Jan. 2007.

[14] B. Vahidi, M. Jannati and S. H. Hosseinian, "A Novel Approach to Adaptive Single Phase Autoreclosure Scheme for EHV Power Transmission Lines Based on Learning Error Function of ADALINE" Simulation, vol. 84, no.12, pp 601-610, 2008.

[15] R. Lawrance and A. Rabiner "A Tutorial on hidden Markov models and selected application in speech recognition", Proc. Of IEEE, vol.77, pp.257-285, 1989.

[16] Jaehak Chung, E. J. Powers, W. M. Grady and S. C. Bhatt "Power disturbance classifier using a rulebased method and wavelet packet-based hidden Markov model", IEEE Trans. Power Del., vol.17, pp. $233-241,2002$.

[17] T. K. Abdel-Galil, A. M. Youssef and M. M. A. Salama "Disturbance Classification Using Hidden Markov Models and Vector Quantization", IEEE Trans. Power Del. vol.20, pp.2129- 2135, 2005.

[18] Abdel-Galil, Y. G. Hegazy, M. M. A Salama and R. Bartnikas "Partial discharge pulse pattern recognition using Hidden Markov Models", IEEE Trans. Dielectrics and Electrical Insulation, vol.11, pp.715 - 723, 2004.

[19] K. C. Kwon and J. H. Kim "Accident identification in nuclear power plants using hidden Markov models", EAAI, vol.12, pp.491-501, 1999.

[20] A. M. Gonzalez, A. M. S. Roque and J.GarciaGonzalez "Modeling and forecasting electricity prices with input/output hidden Markov models", IEEE Trans Power Syst., vol.20, pp.13-24, Jan. 2005.

[21] W. Yu and G. B. Sheblé "Modeling electricity markets with hidden Markov model", EPSR, vol.76, pp.445451, 2006.

[22] M. R. Hassan, B. Nath and M. Kireley "A fusion model of HMM, ANN and GA for stock market forecasting", ESA, vol.33, pp.171-180, 2007.

[23] Qian Suxiang, Jiao Weidong, Hu Hongsheng and Yan Gongbiao, "Transformer Power Fault Diagnosis System Design Based on the HMM Method", IEEE International Conference on Automation and Logistics, pp.1077-1082, 2007.

[24] X. Ma and J. Shi "A new method for discrimination between fault and magnetizing inrush current using HMM", EPSR, vol.56, pp.43-49, 2000.

[25] S. Jazebi, B. Vahidi and S. H. Hosseinian, "A Novel Discriminative Approach Based on Hidden Markov Models and Wavelet Transform to Transformer 
Protection", Simulation, vol.86, no.2, pp.93-107, 2010.

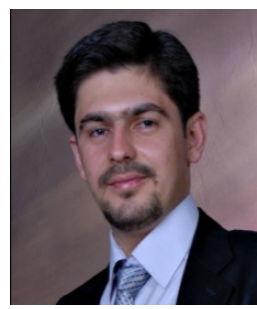

Mohsen Jannati (S'10) received his B.S. degree in Electrical Engineering from Isfahan University of Technology, Isfahan, Iran, in 2006 and his M.S. degree in Electrical Engineering from the Department of Electrical Engineering of Amirkabir University of Technology (AUT), Tehran, Iran, in 2008. He is currently pursuing his Ph.D. degree from the Department of Electrical Engineering at AUT, Tehran, Iran.

His research interests include intelligent fault management and fault diagnosis of power distribution and transmission network, digital protection in power smart grid, and modern power system modeling.

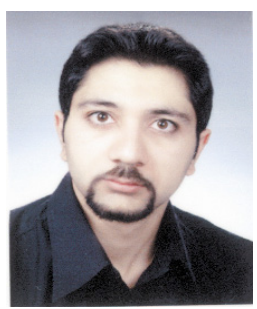

Saeed Jazebi, born in 1983, received his B.S. degree in Electrical Engineering from Shahid Bahonar University, Kerman, Iran, and his M.S. degree at Amirkabir University of Technology, Tehran, Iran. He is now a P.h.D student at the Department of Electrical Engineering in Amirkabir University of Technology, Tehran, Iran. His field of interest includes distribution system operation and planning, optimization techniques, FACTS devices, and application of statistical pattern recognition in the transient analysis of power systems.

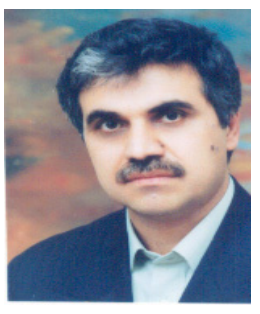

Behrooz Vahidi, born in Abadan, Iran, in 1953, received his B.S. degree in Electrical Engineering from Sharif University of Technology, Tehran, Iran, in 1980 and his M.S. degree in Electrical Engineering from Amirkabir University of Technology, Tehran, Iran, in 1989 . He also received his Ph.D. in Electrical Engineering from UMIST, Manchester, UK, in 1997. He worked in the field of high voltage in the industry as a chief engineer from 1980 to 1986 . From 1989 to present, he has been with the Department of Electrical Engineering of Amirkabir University of Technology, where he is currently a professor. Prof. Vahidi is an IEEE senior member. His main fields of research are high voltage, electrical insulation, power system transient, lightning protection, and pulse power technology. He has authored and co-authored more than 220 papers and 5 books on high voltage engineering and power system.

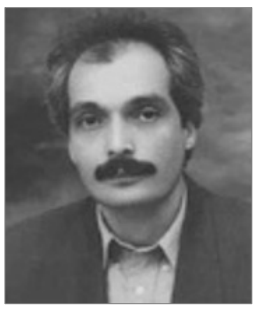

Seyed Hossein Hosseinian, born in 1961 in Iran, received both his B.S. and M.S. degrees from the Electrical Engineering Department of Amirkabir University of Technology (AUT), Iran, in 1985 and 1988, respectively, and his Ph.D. degree from the Electrical Engineering Department of University of Newcastle England in 1995. At present, he is an associate professor at the Electrical Engineering Department of AUT. His special fields of interest include transient in power systems, power quality, and restructuring and deregulation in power systems. 